\title{
A Simple Method for Direct Detection and Discrimination of $A$. baumannii in Tracheal Aspirates without Culture Isolation
}

\section{Cheguri H. Swathi', Sudhaharan Sukanya'2, Vemu Lakshmi2,3, Kamaraju Saipriya1, Venkataraman Sritharan 1,4}

\author{
${ }^{1}$ Department of Molecular Diagnostics \& Biomarkers, Global Medical Education \& Research Foundation (GMERF), \\ Hyderabad, India \\ ${ }^{2}$ Nizam's Institute of Medical Sciences (NIMS), Hyderabad, India \\ ${ }^{3}$ Kamineni Academy of Medical Sciences and Research Center, Hyderabad, India \\ ${ }^{4}$ Department of Molecular Diagnostics \& Biomarkers, Gleneagles Global Hospital, Hyderabad, India \\ Email: swathibiotech511@gmail.com,sukanyavimala@gmail.com, lakshmi57vemu@gmail.com, \\ saipriyatadishetty21@gmail.com, venkataraman.sritharan@gmail.com
}

How to cite this paper: Swathi, C.H., Sukanya, S., Lakshmi, V., Saipriya, K. and Sritharan, V. (2020) A Simple Method for Direct Detection and Discrimination of $A$. baumannii in Tracheal Aspirates without Culture Isolation. Advances in Infectious Diseases, 10, 148-159.

https://doi.org/10.4236/aid.2020.102012

Received: May 15, 2020

Accepted: June 15, 2020

Published: June 18, 2020

Copyright $\odot 2020$ by author(s) and Scientific Research Publishing Inc. This work is licensed under the Creative Commons Attribution International License (CC BY 4.0).

http://creativecommons.org/licenses/by/4.0/

(c) (i) Open Access

\begin{abstract}
Currently, available phenotyping and commercial methods report $A$. baumannii only as Acinetobacter calcoaceticus-baumannii complex (ACB) and do not identify individual members of the complex. This is a single blind study aimed to evaluate certain commonly used species-specific genetic markers namely, Intergenic Transcribed Spacer region in $16 \mathrm{~S}$ rRNA of $A$. baumannii (Ab-ITS) and gyrB, for identification of ACB members. These molecular targets were first validated on clinical isolates $(\mathrm{n}=200)$ and subsequently on uncultured tracheal aspirates $(\mathrm{n}=172)$. Among the clinical isolates, 183/200 (91.5\%) were positive for Ab-ITS. The clinical isolates 17 (17/200) which are failed to amplify in Ab-ITS PCR were subsequently diagnosed by gyrB PCR as $A$. calcoaceticus $(\mathrm{n}=2), A$. pitti $(\mathrm{n}=6)$ and $A$. nosocomialis $(\mathrm{n}=9)$ but not $A$. baumannii. Among the tracheal aspirates, 62 samples were reported as sterile in Advanced Expert System of VITEK-2, among the remaining 110 samples, 68.1\% (75/110) samples contained AbITS target. Twenty-five of the sterile samples (25/62) were found to contain $\mathrm{Ab}$-ITS target sequence. Since, our sample processing method enabled identification of all the species of ACB complex by PCR even in uncultured tracheal aspirates, adaptation of our protocol would enable same day (6 - $8 \mathrm{~h}$ ) reporting and help the clinician make evidence based therapeutic decision quickly.
\end{abstract}




\section{Keywords}

Acinetobacter baumannii, ACB Complex, Ab-ITS, gyrB, PCR, Tracheal Aspirates

\section{Introduction}

Acinetobacter baumannii causes a vast array of infections including ventilator associated pneumonia (VAP), secondary meningitis, urinary tract infections, septicemia and other severe infectious complications in the critical care (CCU) patients [1] [2]. Clinical microbiology laboratories usually report $A$. baumannii as "ACB complex". This includes six individual species namely $A$. baumannii, $A$. calcoaceticus, $A$. pitti, and A. nosocomialis, A. seifertii, A. lactucae [3]. These members have epidemiological and clinically relevant differences. They exhibit different drug susceptibility profiles though they are genetically and phenotypically very similar. In particular, $A$. baumannii is responsible for a large proportion of nosocomial infections. There has been a tremendous increase in antibiotic resistance among $A$. baumannii isolates in the last two decades [4]. Members of ACB complex pose limitations in phenotyping and conventional microbiological identification [5] [6] [7] [8]. Therefore, it would be desirable to apply molecular methods to identify the individual species of the ACB complex [7] [9]. Also, it would be ideal to be able to detect and report the species directly rapidly from the clinical sample without waiting for culture isolation and characterization.

Molecular methods which are used for identification of Acinetobacter spp. include ribotyping, polymerase chain reaction (PCR) hybridization with speciesspecific probes, methods based on the $16 \mathrm{~S}$ - 23S rRNA intergenic spacer region, tRNA spacer fingerprinting, Pulse Field Gel Electrophoresis (PFGE), methods based on $g y r B$ and $r p o B$ sequences, PCR-RFLP and AFLP analysis, Amplified Ribosomal DNA Restriction Analysis (ARDRA) and Random Amplified Polymorphic DNA (RAPD) typing [10] [11]. Amplified Ribosomal DNA Restriction Analysis (ARDRA) is rapid and more practical for species identification [12]. The main disadvantage of these typing methods is low reproducibility, especially in terms of global molecular epidemiology [7]. Sequence-based typing, such as single or multi locus or whole-genome sequencing, provides more reliable data for comparison from a geographical point of view or globally. However, the high cost of sequence-based typing prevents implementation by developing countries [6] [13]. Failure to identify the A. baumannii in ACB complex may adversely impact both diagnosis and infection control. Thus, there is an unmet need for a rapid and affordable diagnostic tool to identify $A$. baumannii in clinical isolates in general and particularly from uncultured clinical specimens. A promising and robust molecular target for identification of Acinetobacter spp. would be the polymorphisms within the rRNA intergenic spacer (ITS) sequences. These rRNA genes are present in the same order (i.e. 16S, 23S, and 5S) in their transcriptional 
units and well conserved throughout most bacterial species [14]. Among the available genetic targets, intergenic spacer in A. baumannii (Ab-ITS) holds-out as a good genetic marker for the species identification as it offers low intra species variation and high degrees of interspecies divergence [1] [9].

A simple sample processing protocol and PCR were optimized for detection of $A$. baumannii in uncultured tracheal aspirates. The internal transcribed spacer region of the ribosomal RNA of $A$. baumannii, called Ab-ITS, was used for identification of $A$. baumannii species. Results of this Ab-ITS-PCR were evaluated against conventional clinical microbiology phenotyping methods and VITEK 2 system. We also present in this article our experience of using gyrB based target sequences for differentiation of individual members of $\mathrm{ACB}$ complex.

\section{Materials and Methods}

This is a prospective study conducted from November 2014 to March 2015, at Gleneagles Global Hospitals.

\subsection{Specimen Collection}

Samples were collected from two tertiary care health centers in Hyderabad for this study.

Category 1: This group comprised of characterized archival clinical isolates which were reported as ACB complex. These isolates were collected from the department of Clinical Microbiology of Gleneagles Global hospital, Hyderabad $(\mathrm{n}=200)$.

Category 2: The leftover tracheal aspirate specimens $(n=172)$ from the department of clinical microbiology of Nizam's Institute of Medical Sciences (NIMS), Hyderabad were collected, and stored at $2^{\circ} \mathrm{C}-8^{\circ} \mathrm{C}$ (not more than $48 \mathrm{~h}$ ) till sample processing was performed for genotyping. No criteria were imposed on inclusion of these samples except that all tracheal aspirates were collected from patients in the intensive care units suffering from pneumonia. The results of clinical microbiology characterization of these tracheal aspirate samples were blinded to our laboratory and were decoded only after the Ab-ITS genotyping was completed.

Four reference strains-A. baumannii (ATCC 17978), A. pitti (MCC 2077-T), A. calcoaceticus (MCC 2329), A. nosocomialis (ATCC 17903) belonging to different genome species of ACB complex were obtained from culture collections (ATCC and National Center for Microbial Resource, India), for quality assurance. Staphylococcus aureus (ATCC 43300, ATCC 25923) was used as negative control.

\subsection{Genomic DNA Preparation}

Cell free DNA lysates were prepared from isolates of Category 1 and tracheal aspirates of Category 2, by Tris-EDTA-Triton-X (TEX) buffer protocol (10 mM Tris- $\mathrm{HCl} \mathrm{pH}$ 8.5, 1 mM EDTA, 1\% (w/v) Triton X-100) [15]. Single colonies 
isolated on the MHA were inoculated into $\mathrm{MH}$ broth and allowed to grow at $37^{\circ} \mathrm{C}$ for $24 \mathrm{~h}$ and processed just like tracheal aspirates. The concentration of DNA (ng/ $\mu$ l) was determined by absorbance (260/280) in Nanodrop ND2000. The sample processing is presented schematically in Figure 1.

\subsection{Genotyping of A. baumannii by Ab-ITS PCR}

PCR assay was performed with the genomic DNA in $20 \mu$ total reaction volume. The PCR reaction mixture contained 20 pmol of each primer, $200 \mu \mathrm{mol}$ of dNTPs, $1 \mathrm{U}$ of Taq DNA polymerase (KAPA Taq DNA Polymerase, KAPA Biosystems Inc), $2 \mu \mathrm{l} 10 \mathrm{X}$ buffer, $1.65 \mathrm{mM} \mathrm{MgCl}_{2}$ and $100 \mathrm{ng}$ of genomic DNA lysate. The primers and PCR conditions used in this study are given in Table 1. The amplicons of ITS region and $g y r B$ were resolved and analyzed on $2 \%$ agarose gel after ethidium bromide staining and documented in a gel documentation system (UVITEC, UK). The tracheal aspirate's phenotyping results were de-coded after the genotyping was completed.

\section{Results}

\subsection{Phenotyping and AST}

The 200 ACB isolates which belonged to Category 1 were identified in the clinical microbiology laboratory using both manual (biochemical) and automated methods and results interpreted as per BSAC guidelines (2015) [16].

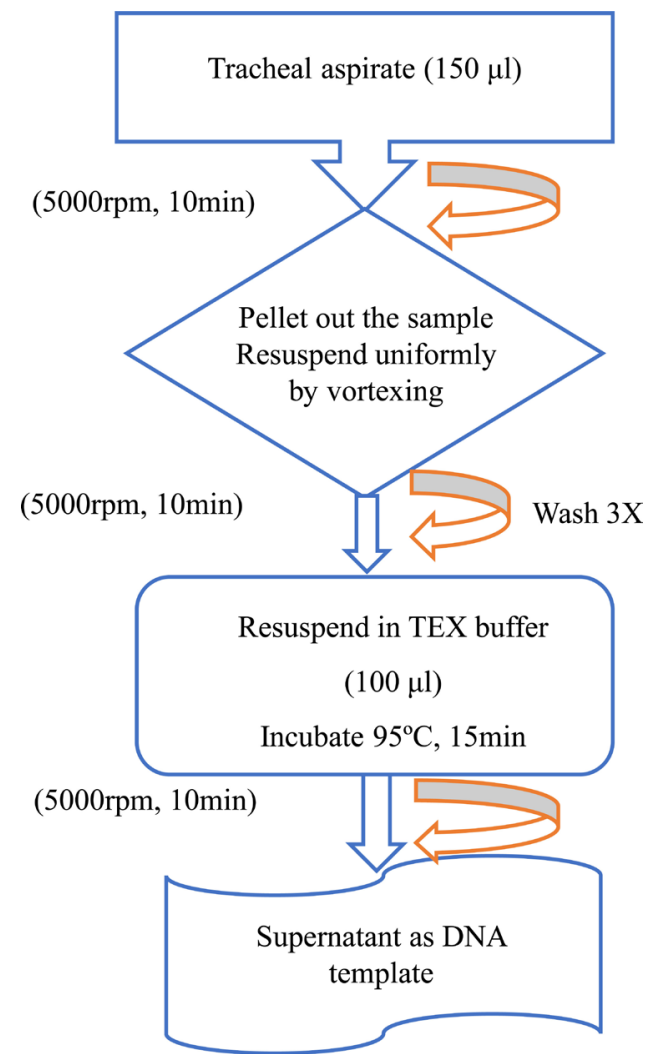

Figure 1. Schematic of tracheal samples processing through TEX method. 
Table 1. List of primers and PCR conditions used in this study.

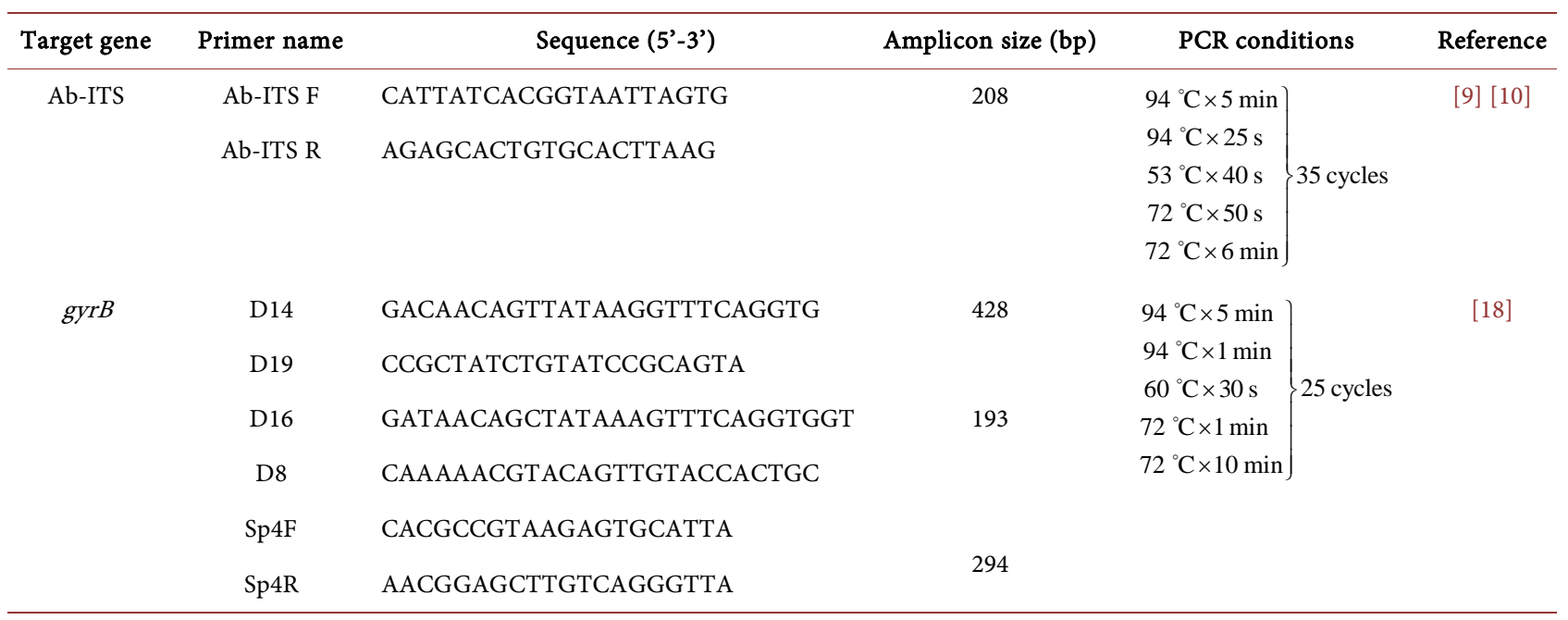

The microbial profiles of all tracheal aspirates were determined using VITEK-2 (BioMérieux, Marcy l'Etoile France) in the clinical microbiology department, as per Clinical \& Laboratory Standards Institute (CLSI, 2015) guidelines (Wayne 2017) [17].

\subsection{Ab-ITS PCR Results}

\section{Category $1(n=200)$ ACB isolates}

$183(91.5 \%)$ isolates were positive for Ab-ITS and a typical pattern of amplicons in agarose gel electrophoresis is represented in Figure 2. Results of identification and drug sensitivity profiles of ACB isolates are listed in Table 2.

\subsection{Characterization of the Tracheal Aspirates}

After phenotyping, 110 tracheal aspirates were reported as: $A$. baumannii (n = $43)$, Polymicrobial growth $(\mathrm{n}=23)$, Colonizers $(\mathrm{n}=20), K$. pneumonia $(\mathrm{n}=8)$, $P$. aeruginosa $(\mathrm{n}=7), E$. coli $(\mathrm{n}=2)$, MRSA $(\mathrm{n}=3)$, Diphtheroids $(\mathrm{n}=2)$ and others $(n=2)$. Sixty-two tracheal aspirates $(36 \%, 62 / 172)$ were reported as sterile by VITEK-2. Among these 172 tracheal aspirates, 90 (52.3\%) were positive for $A$. baumannii specific marker (Ab-ITS). Among the 62 samples which were reported as sterile by Advanced Expert System of VITEK-2, twenty-five (25/62) were positive for Ab-ITS. Decoded phenotypic data of tracheal aspirates in comparison to our PCR are presented in Table 3.

\subsection{Genotyping of ACB Complex by gyrB PCR}

Several isolates (17/200) of Category 1 tested negative for Ab-ITS PCR. Since gyrB PCR has been successfully applied to discriminate and identify members of ACB complex [9] [10] [18]. We first optimized the amplification conditions for the four members of ACB complex: they were $A$. calcoaceticus, $A$. pitti and $A$. nosocomialis in addition to $A$. baumannii and interestingly all the seventeen of them were identified as $A$. calcoaceticus $(\mathrm{n}=2), A$. pitti $(\mathrm{n}=6)$ and $A$. nosocomialis 


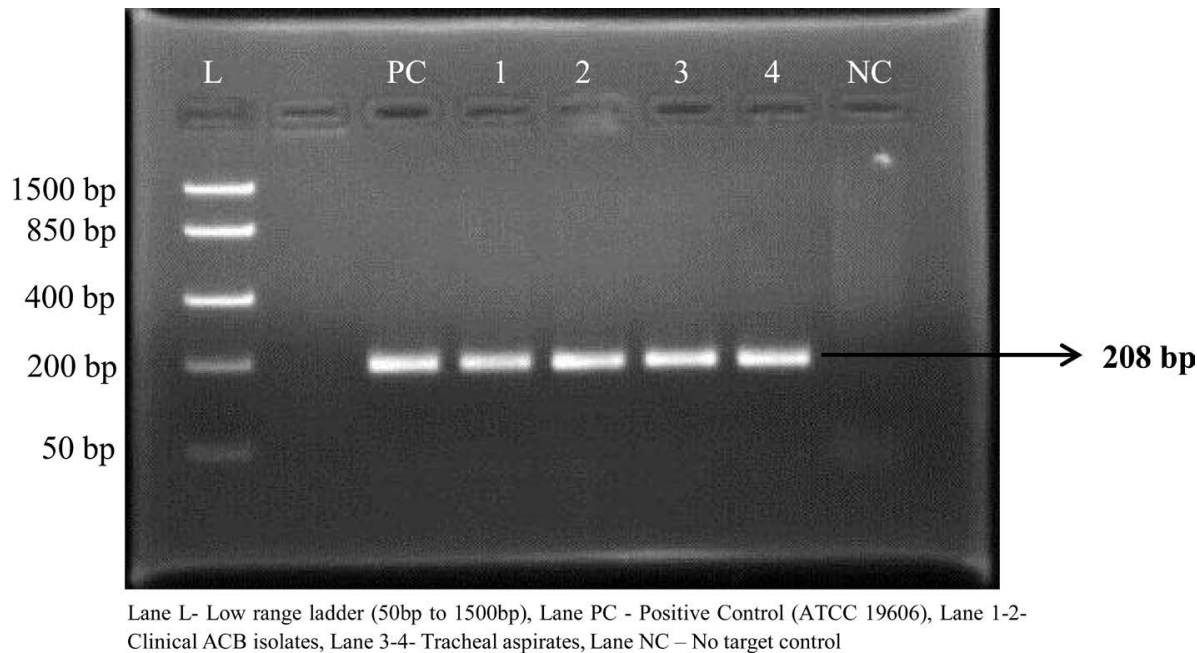

Figure 2. Agarose gel electrophoresis of Ab-ITS PCR.

Table 2. Performance of genotyping and phenotyping approaches used in the identification of ABC isolates.

\begin{tabular}{ccccc}
\hline S. No. & Sample Source & Ab-ITS $^{*}(\mathrm{n})$ & $\operatorname{gyr}^{*}(\mathrm{n})$ & ACB members $(\mathrm{n})$ \\
\hline 1 & Respiratory secretions (73) & 69 & 4 & A. baumannii $(\mathrm{n}=69)$, A. nosocomialis $(\mathrm{n}=2)$, A. pitti $(\mathrm{n}=2)$ \\
2 & Blood (50) & 47 & 3 & A. baumannii $(\mathrm{n}=47)$, A. nosocomialis $(\mathrm{n}=1)$, A. pitti $(\mathrm{n}=2)$ \\
3 & Wound swab (38) & 33 & 5 & A. baumannii $(\mathrm{n}=33)$, A. nosocomialis $(\mathrm{n}=3)$, A. pitti $(\mathrm{n}=2)$ \\
4 & Sputum (33) & 29 & 4 & A. baumannii $(\mathrm{n}=29)$, A. nosocomialis $(\mathrm{n}=3)$, A. calcoaceticus $(\mathrm{n}=1)$ \\
5 & Body fluids (6) & 5 & 1 & A. baumannii $(\mathrm{n}=5)$, A. calcoaceticus $(\mathrm{n}=1)$ \\
& Total (200) & 183 & 17 & \\
\hline
\end{tabular}

*Species specific marker (Ab-ITS); "gyrase B gene (DNA topoisomerase); $\mathrm{n}=$ Number of positives.

Table 3. Decoded phenotypic data of tracheal aspirates in comparison to our PCR genotyping.

\begin{tabular}{ccc}
\hline Microbial ID from Tracheal aspirates & VITEK-2 Results (n) & Ab-ITS * Results (n) \\
\hline A. baumannii & 43 & 39 \\
E. Coli & 2 & 0 \\
Klebsiella pneumonia & 8 & 0 \\
Pseudomonas aeruginosa & 7 & 0 \\
Diphtheroids & 2 & 1 \\
Serratiamarcescens + A. baumannii & 1 & 0 \\
MRSA & 3 & 11 \\
${ }^{\text {aPolymicrobial growth }}$ & 21 & 2 \\
${ }^{\mathrm{c}}$ Colonizers with $A$. baumannii & 2 & 2 \\
${ }^{\mathrm{d}}$ Colonizers without $A$. baumannii & 2 & 9 \\
${ }^{\mathrm{e}}$ No growth & 18 & 25 \\
TOTAL & $62($ Sterile $)$ & $90^{*}$
\end{tabular}

*-Ab-ITS-16S-23S rRNA gene intergenic spacer region, ${ }^{\mathrm{a}, \mathrm{b}, \mathrm{b}, \mathrm{d}, \mathrm{e}}$ - Results as obtained from Advanced Expert System of VITEK-2, ${ }^{*}-$ Total number of Ab-ITS positive samples (including sterile samples). 
$(\mathrm{n}=9)$. The results are documented in Figure 3.

Four of the tracheal isolates were reported as $\mathrm{ACB}$ complex but when we screened them by Ab-ITS PCR, they did not contain the Ab-ITS target sequence $(\mathrm{n}=4)$. These tracheal aspirates were processed as shown in scheme Figure 1 and subjected to gyr $B$-PCR with the primer sets for the above mentioned four species of ACB complex and analyzed by agarose gel electrophoresis to identify the species (Figure 4). Two of them were identified as A. nosocomialis $(\mathrm{n}=2)$ and we could not confirm the Acinetobacter species of the other two samples.

\subsection{Statistical Analysis of the Performance of PCR Assays}

MedCalc software (MedCalc Statistical Software version 15.6.1, MedCalc Software,

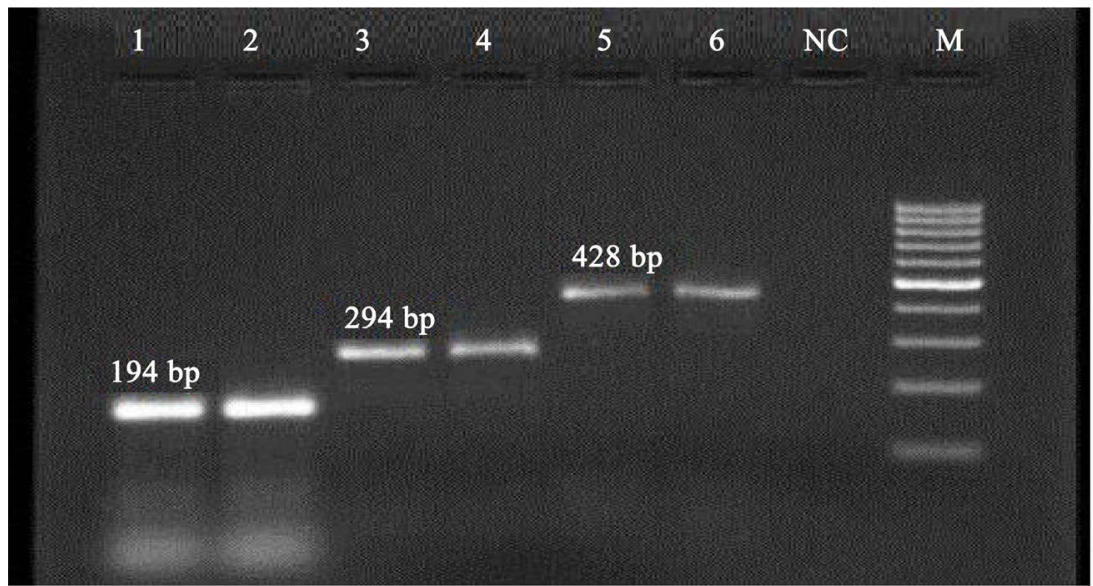

Lane M- Molecular marker (100bp to 1000bp), Lane 1- Positive Control (A. pitti MCC 2397), Lane 2, 4 \& 6 - ACB clinical isolates, Lane 3 - Positive Control (A. nosocomialis ATCC 17903), Lane 5 -Positive Control (A. calcoaceticus MCC 2077(T), Lane NC - No target control

Figure 3. gyrB genotyping of ACB complex by PCR.

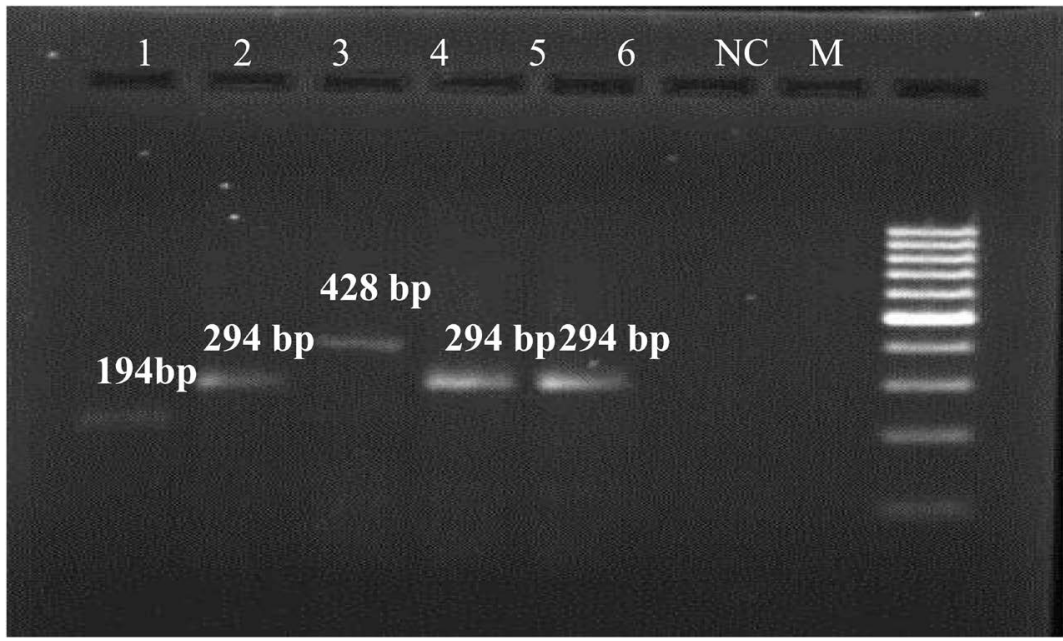

Lane M-Molecular marker (100bp to 1000bp) Lane 1-Positive Control (A. pitti MCC 2397), Lane 2-Positive Control (A. nosocomialis ATCC 17903), Lane 3-Positive Control (A. calcoaceticus MCC 2077(T), Lane 4 \& 5 -Tracheal aspirates positive for $A$. nosocomialis, Lane 6-Tracheal aspirate negative for ACB members. Lane NC-Negative PCR Control.

Figure 4. gyrB PCR results of tracheal aspirates. 
Ostend, Belgium; https://www.medcalc.org; 2015) was used for statistical analysis of the data.

The Ab-ITS PCR had $92 \%$ Sensitivity, 100\% Specificity, with a Positive Predictive Value (PPV) of $98.92 \%$ and $100 \%$ Negative Predictive Value (NPV) (Figure $5)$.

When we evaluated the performance of the genetic marker Ab-ITS against the reference method VITEK-2, 44(44/110) tracheal aspirates were positive by both PCR and VITEK-2, whereas 31 samples were "false positive" in PCR genotyping. Thus Ab-ITS PCR showed greater sensitivity (100\%) and specificity (53.03\%) compared to VITEK-2 for identification of Acinetobacter baumannii (Figure 6).

\section{Discussion}

A. baumannii has emerged as one of the most notorious pathogens in health care facilities globally, targeting the critically ill hospitalized patients with its dreadful drug resistance profile. With an MDR rate of $92.1 \%$ and no new drug on the horizon, $A$. baumannii infections have become a real challenge for critical care and general physicians [7] [19] [20] [21]. Reporting time for identification of $A$. baumannii in clinical samples directly impacts the outcome of critical care. In this study we have focused on the identification of $A$. baumannii by evaluating a commonly used species-specific genetic marker and subsequently using a simple sample processing protocol for tracheal aspirates coupled to PCR for direct detection

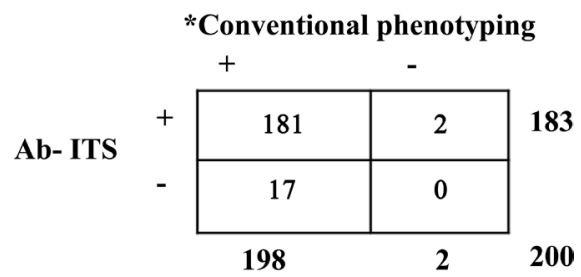

Statistical analysis: Sensitivity 92\%, Specificity 100\%, Positive Predictive Value (PPV) 98.92\% and Negative Predictive Value 100\% (MedCalc Statistical Software version 15.6.1). ${ }^{\star}$ Species identification and characterization done by biochemical tests.

Figure 5. Comparison of species-specific marker (Ab-ITS) vs Phenotypic assay in ACB isolates $(n=200)$.

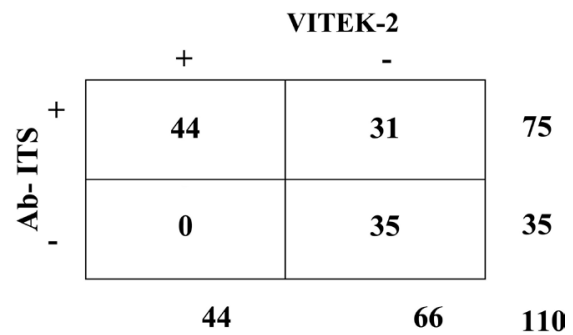

Statistical analysis: Sensitivity 100\%, Specificity 53.03\%, Positive Predictive Value (PPV) 58. 67\% and Negative Predictive Value (NPV) 100\% (MedCalc Statistical Software version 15.6.1). This analysis considered only the clinical samples contained bacterial growth (110/172) and 62 samples were sterile samples.

Figure 6. Comparison of species-specific marker (Ab-ITS) vs VITEK-2 in tracheal aspirates $(\mathrm{n}=110)$. 
and confirmation of $A$. baumannii in uncultured clinical sample. It is well known that tracheal aspirates are rich source of mixed microbes. Our protocol worked remarkably well on these samples and was able to identify $A$. baumannii correctly. Ab-ITS seems to be a more reliable and sensitive genetic marker for identification of $A$. baumannii. Sequences of the ITS regions have been found to have low levels of intra-species variation and high levels of interspecies divergence [9] [10]. We used the primer sequences of Chen et al. (2007) and it was interesting to note that these primers proved absolutely specific to $A$. baumannii [10]. Including species specific gyrB primers along with Ab-ITS primers would be an ideal way to make differential diagnosis of ACB complex directly from uncultured clinical samples. Acinetobacter genus consists of about 33 species and six of them, grouped as ACB, are more frequently associated with human diseases. They are A. baumannii, Acinetobacter 13TU and Acinetobacter genome species 3 were frequently found in several outbreaks [7]. Commercial systems like API 20NE and VITEK-2 are increasingly being used for identification of species but these systems fail to discriminate closely related species [22]. The six members of ACB complex are very closely related and difficult to differentiate by conventional phenotyping or as was shown in this study even by automated systems like VITEK 2 and are missed sometimes even by PCR. Individual members of ACB complex should be identified correctly from $A$. baumannii as their drug sensitivity profiles are different [5]. Ab-ITS PCR is a reliable, accurate method and can be applied directly on uncultured tracheal aspirates for rapid identification of $A$. baumannii. The sample processing protocol used in this study is derived from the original protocol published decades ago for processing a variety of clinical samples for the detection of $M$. tuberculosis and therefore, it should work well with any type of clinical specimen [19]. However, since the microbial population would be different in each type of specimen, our protocol is currently being validated on other types of clinical specimen.

The combination of genetic markers used in this study, provides powerful information not only on identification of $A$. baumannii but also on probable outbreak potential and allows, to some extent, prediction of carbapenem resistance. The superiority of our genotyping approach compared to VITEK-2 is evident from the sensitivity profile and helps significantly in the differential diagnosis of individual members of ACB complex. Further, our protocol can be applied to both clinical isolates and for direct detection from uncultured clinical samples. This is certainly an advantage as results could be intimated to clinician the same day to make quick therapeutic decisions. Sixty-two tracheal aspirates were reported as sterile by VITEK-2 (mainstay in many advanced clinical microbiology laboratories) while twenty-five of them found to harbor Ab-ITS target sequence specific for A. baumannii. Availability of multiple copies of 16S-23S rRNA in bacterial cells ensures superior sensitivity of PCR compared to culture. It is possible that these patients apparently had $A$. baumannii infection and the antimicrobial therapy killed them. Such information from the "so called sterile sam- 
ples" will be very useful for transmission, antibiotic therapy and molecular epidemiology. Our method has not yet been validated on $A$. seifertii, A. lactucae which are also members of ACB complex and further we have demonstrated our method only on clinical isolates and tracheal aspirates. We are hopeful that the sample processing protocol would work equally well with all types of clinical specimen as it was originally reported [15]. The in-house sample processing and PCR methods for clinical specimen can be easily adopted for rapid detection of A. baumannii much before culture results are obtained. Implementation of this simple and economical protocol would help the clinician to make evidence based therapeutic decision on the same day.

\section{Conclusion}

This study showed that the $16 \mathrm{~S}$ - 23S rRNA intergenic spacer region possesses high discriminatory and better sensitivity to identify $A$. baumannii to the species level among ACB complex compared to conventional microbiology techniques. In this study, a comparison between a well-established species-specific genetic marker, Ab-ITS and a commonly used microbial identification system VITEK 2, was made. A convenient and rapid sample processing method was evaluated, for identification of individual species of $\mathrm{ACB}$ complex and their drug resistance profiles. The results are available in about $6-8 \mathrm{~h}$, enabling the clinicians in infection control. Implementation of this protocol would help not only in control of hospital acquired infections but also improve the outcome of such infections.

\section{Acknowledgements}

Authors thank Clinical Microbiology department of Gleneagles Global Hospitals for kindly providing characterized clinical isolates of $\mathrm{ACB}$ complex for this study.

\section{Conflicts of Interest}

The authors declare no conflicts of interest regarding the publication of this paper.

\section{References}

[1] Chuang, Y.C., Sheng, W.H., Li, S.Y., Lin, Y.C., Wang, J.T. and Chen, Y.C. (2011) Influence of Genospecies of Acinetobacter baumannii Complex on Clinical Outcomes of Patients with Bacteremia. Clinical Infectious Diseases, 52, 352-360. https://doi.org/10.1093/cid/ciq154

[2] Bergogne-Berezin, E. and Towner, K.J. (1996) Acinetobacter spp as Nosocomial Pathogens: Microbiological, Clinical, and Epidemiological Features. Clinical Microbiology Reviews, 9, 148-165. https://doi.org/10.1128/CMR.9.2.148-165.1996

[3] Park, Y.K., Jung, S.I., Park, K.H., Kim, D.H., Choi, J.Y., Kim, S.H. and Ko, K.S. (2012) Changes in Antimicrobial Susceptibility and Major Clones of Acinetobacter calcoaceticus-baumannii Complex Isolates from a Single Hospital in Korea over 7 Years. Journal of Medical Microbiology, 61, 71-79. 
https://doi.org/10.1099/jmm.0.033852-0

[4] Abbo, A., Navon-Venezia, S., Hammer-Muntz, O., Krichali, T., Siegman-Igra, Y. and Carmeli, Y. (2005) Multidrug-Resistant Acinetobacter baumannii. Emerging Infectious Diseases, 11, 22-29. https://doi.org/10.3201/eid1101.040001

[5] Seifert, H., Baginski, R., Schulze, A. and Pulverer, G. (1993) Antimicrobial Susceptibility of Acinetobacter Species. Antimicrobial Agents and Chemotherapy, 37, 750-753. https://www.ncbi.nlm.nih.gov/pubmed/8494371 https://doi.org/10.1128/AAC.37.4.750

[6] Ko, W.C., Lee, N.Y., Su, S.C., Dijkshoorn, L., Vaneechoutte, M., Wang, L.R., et al. (2008) Oligonucleotide Array-Based Identification of Species in the Acinetobacter calcoaceticus- $A$. baumannii Complex in Isolates from Blood Cultures and Antimicrobial Susceptibility Testing of the Isolates. Journal of Clinical Microbiology, 46, 2052-2059. https://doi.org/10.1128/JCM.00014-08

[7] Peleg, A.Y., Seifert, H. and Paterson, D.L. (2008) Acinetobacter baumannii: Emergence of a Successful Pathogen. Clinical Microbiology Reviews, 21, 538-582.

https://doi.org/10.1128/CMR.00058-07

[8] Pantophlet, R., Severin, J.A., Nemec, A., Brade, L., Dijkshoorn, L. and Brade, H. (2002) Identification of Acinetobacter Isolates from Species Belonging to the Acinetobacter calcoaceticus-Acinetobacter baumannii Complex with Monoclonal Antibodies Specific for O Antigens of Their Lipopolysaccharides. Clinical and Vaccine Immunology, 9, 60-65. https://doi.org/10.1128/CDLI.9.1.60-65.2002

[9] Chang, H.C., Wei, Y.F., Dijkshoorn, L., Vaneechoutte, M., Tang, C.T. and Chang, T.C. (2005) Species-Level Identification of Isolates of the Acinetobacter calcoaceticus-Acinetobacter baumannii Complex by Sequence Analysis of the 16S-23S rRNA Gene Spacer Region. Journal of Clinical Microbiology, 43, 1632-1639. https://doi.org/10.1128/JCM.43.4.1632-1639.2005

[10] Chen, T.L., Siu, L.K., Wu, R.C., Shaio, M.F., Huang, L.Y. and Fung, C.P. (2007) Comparison of One-Tube Multiplex PCR, Automated Ribotyping and Intergenic Spacer (ITS) Sequencing for Rapid Identification of Acinetobacter baumannii. Clinical Microbiology and Infection, 13, 801-806. https://doi.org/10.1111/j.1469-0691.2007.01744.x

[11] Yamamoto, S. and Harayama, S. (1996) Phylogenetic Analysis of Acinetobacter Strains Based on the Nucleotide Sequences of $g y r \mathrm{~B}$ Genes and on the Amino Acid Sequences of Their Products. International Journal of Systematic and Evolutionary Microbiology, 46, 506-511. https://doi.org/10.1099/00207713-46-2-506

[12] Vaneechoutte, M., Dijkshoorn, L., Tjernberg, I., Elaichouni, A., De Vos, P., Claeys, G. and Verschraegen, G. (1995) Identification of Acinetobacter Genomic Species by Amplified Ribosomal DNA Restriction Analysis. Journal of Clinical Microbiology, 33, 11-15. https://www.ncbi.nlm.nih.gov/pubmed/7699025 https://doi.org/10.1128/JCM.33.1.11-15.1995

[13] Bartual, S.G., Seifert, H., Hippler, C., Luzon, M.A., Wisplinghoff, H. and Rodriguez Valera, F. (2005) Development of a Multilocus Sequence Typing Scheme for Characterization of Clinical Isolates of Acinetobacter baumannii. Journal of Clinical Microbiology, 43, 4382-4390. https://doi.org/10.1128/JCM.43.9.4382-4390.2005

[14] Dolzani, L., Tonin, E., Lagatolla, C., Prandin, L. and Monti Bragadin, C. (1995) Identification of Acinetobacter Isolates in the A. calcoaceticus- $A$. baumannii Complex by Restriction Analysis of the 16S-23S rRNA Intergenic-Spacer Sequences. Journal of Clinical Microbiology, 33, 1108-1113.

https://www.ncbi.nlm.nih.gov/pmc/articles/PMC228114/ 
https://doi.org/10.1128/JCM.33.5.1108-1113.1995

[15] Sritharan, V. and Barker, R.H. (1991) A Simple Method for Diagnosing M. tuberculosis Infection in Clinical Samples Using PCR. Molecular and Cellular Probes, 5, 385-395. https://doi.org/10.1016/S0890-8508(06)80011-3

[16] BSAC. BSAC Methods for Antimicrobial Susceptibility Testing. http://www.bsac.org.uk/wp-content/uploads/2012/02/BSAC-disc-susceptibility-testi ng-methods-Jan-2015.pdf

[17] Clinical and Laboratory Standards Institute (2017) Performance Standards for Antimicrobial Susceptibility Testing: 27th Informational Supplement M-100-S27. Wayne.

[18] Paul, G.H., Marlene, L., Hilmar, W. and Harald, S. (2010) gyrB Multiplex PCR to Differentiate between Acinetobacter calcoaceticus and Acinetobacter Genomic Species 3. Journal of Clinical Microbiology, 48, 4592-4594.

https://doi.org/10.1128/JCM.01765-10

[19] Heritier, C., Poirel, L., Fournier, P.E., Claverie, J.M., Raoult, D. and Nordmann, P. (2005) Characterization of the Naturally Occurring Oxacillinase of Acinetobacter baumannii. Antimicrobial Agents and Chemotherapy, 49, 4174-4179.

https://doi.org/10.1128/AAC.49.10.4174-4179.2005

[20] Perez, F., Hujer, A.M., Hujer, K.M., Decker, B.K., Rather, P.N. and Bonomo, R.A. (2007) Global Challenge of Multidrug Resistant Acinetobacter baumannii. Antimicrobial Agents and Chemotherapy, 51, 3471-3484. https://doi.org/10.1128/AAC.01464-06

[21] Zivanovic, V., Goikovic-Bukarica, L., Scepanovic, R., Vitotovic, T. and Novakovic, R. (2017) Differences in Antimicrobial Consumption, Prescribing and Isolation Rate of Multidrug Resistant Klebsiella pneumoniae, Pseudomonas aeruginosa and Acinetobacter baumannii on Surgical and Medical Wards. PLoS One, 12, e0175689. https://doi.org/10.1371/journal.pone.0175689

[22] Zavascki, A.P., Carvalhaes, C.G., Picao, R.C. and Gales, A.C. (2010) Multidrug-Resistant Pseudomonas aeuroginosa and Acinetobacter baumannii: Resistance Mechanisms and Implications for Therapy. Expert Review of Anti-infective Therapy, 8 , 71-93. https://doi.org/10.1586/eri.09.108 